\title{
Characterization of diesel particulate collected with ELPI by HRTEM
}

\author{
D. Berti, ${ }^{*}$ D. Faedo** and F. Avella** \\ * EniTecnologie, Dipartimento di Chimica Fisica, Via Maritano 26, 20097 San Donato Milanese \\ (MI), Italy \\ ** Stazione Sperimentale Combustibili, Viale De Gasperi 16, 20097 San Donato Milanese (MI), \\ Italy
}

$\mathrm{PM}_{2.5}$ is the particulate matter with aerodynamic diameter lower than $2.5 \mu \mathrm{m}$; it is mainly produced by combustion processes (vehicle traffic, heating, industrial plants, etc.) and by gas-to-particle conversion processes during dilution of exhaust gases.

In the last years $\mathrm{PM}_{2.5}$ has been widely studied because of its negative effects on health ${ }^{1}$ but there is still little information on its physicochemical properties.

Due to its dimensions, TEM (Transmission Electron Microscopy) coupled with EDS (Energy Dispersive Spectroscopy) is a very suitable method for its characterization ${ }^{2}$.

The aim of this work was to investigate morphology and composition of $\mathrm{PM}_{2.5}$ produced by diesel vehicles with different fuels and at different working conditions, with a particular attention for its smallest fraction $(<100 \mathrm{~nm})$.

The observations were done with a High Resolution Transmission Electron Microscope (Jeol JEM 3010: acceleration voltage $300 \mathrm{kV}$; resolution $1.7 \AA$ ) equipped with EDS microanalysis (Link Isis 200ATW, detecting elements from C) and a CCD camera for digital image acquisition. Dimensional ranges were determined by manual image analysis (IA).

In order to collect the soot without introducing artefacts and to conserve its original morphology and composition, on-line sampling was used ${ }^{3}: 3 \mathrm{~mm}$ diameter copper support grids were deposited on paper filters and the particulate was collected directly upon them from the tailpipe, with short collecting times (1-10 minutes) and without any manipulation. This procedure is a very good alternative to thermophoretic sampling, that requires a very expensive apparatus ${ }^{4}$.

To separate the different sizes before the observation, $\mathrm{PM}_{2.5}$ was collected from ELPI (Electric Low Pressure Impactor) plates with a special procedure.

Samples from different sources (EuroI and EuroII common rail engines) and fuels (diesel, biodiesel, etc.), taken at different speeds $(32 \mathrm{~km} / \mathrm{h}, 120 \mathrm{~km} / \mathrm{h})$, were examined. All of them have the same basic structure: $\mathrm{PM}_{2.5}$ is formed by aggregates of small, amorphous elementary particles. They are mostly spherical and contain only carbon. The dimensions vary in the range 10-20 nm. With the new lowemissions engines, their size and composition are not significantly affected by changing the fuel or the operating conditions.

The aggregates, 0.1-3 $\mu \mathrm{m}$ in size, have bizarre shapes: they can be monodimensional (like chains or branches), bidimensional (like flakes) or tridimensional (like sponges). They are made only by elementary particles and do not have any condensation nucleus inside. The aggregate shapes vary with their dimensions (Figure 1-3). If metal-containing additives are added in the fuel, they form oxide particles, separated from the carbonaceous ones (Figure 4). 
References

[1] L.M. Brown et al., Phil. Trans. R. Soc. Lond. A 358, (2000) 2563

[2] A.D. Maynard, Phil. Trans. R. Soc. Lond. A 358 (2000) 2593

[3] K.A. Berubè et al., Atmos. Environment 33 (1999) 1599

[4] U.O. Koiluet al., Combustion and Flame (1997) 110494
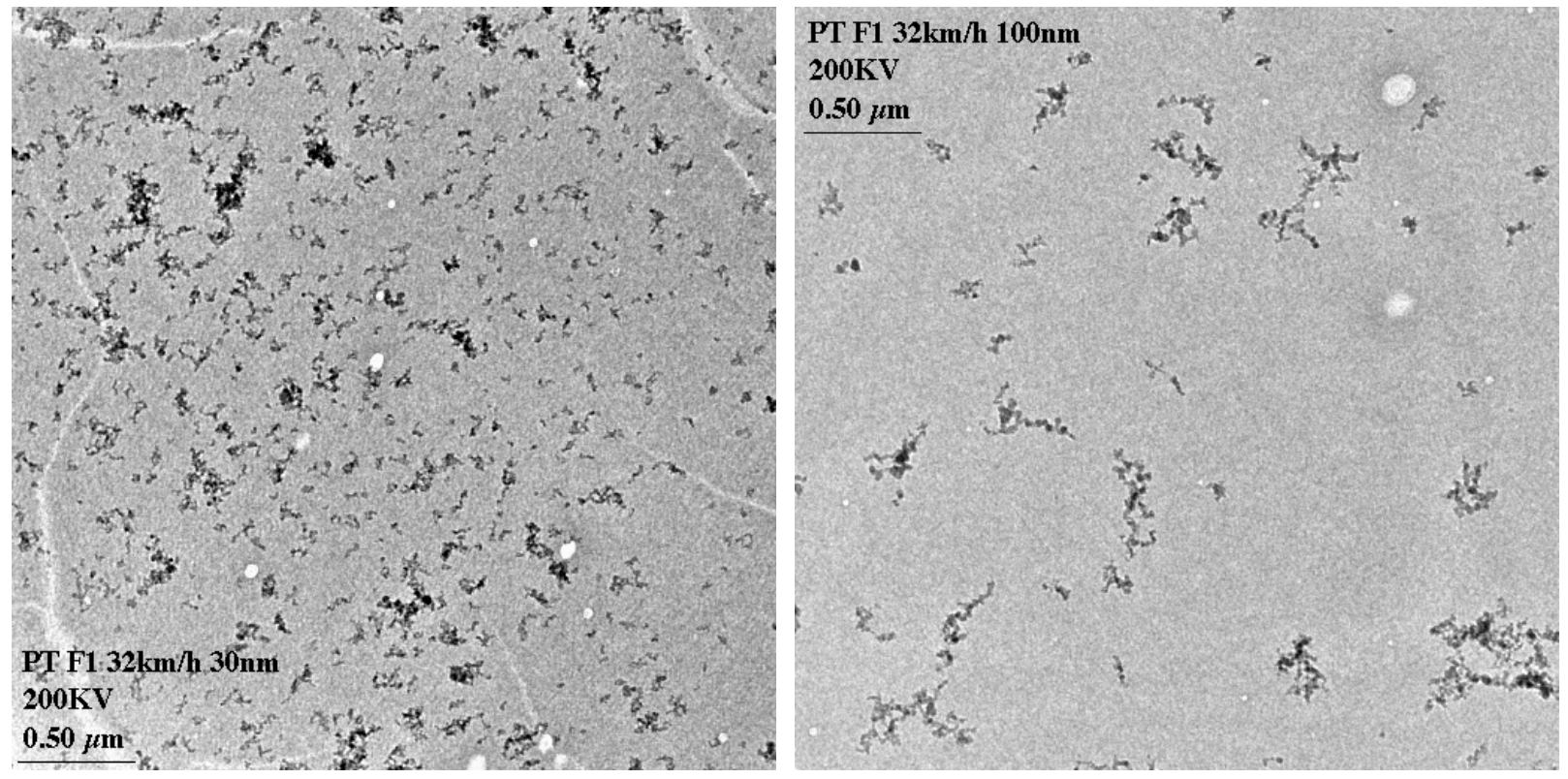

FIG. 1. PM collected on ELPI stage $1(30-60 \mathrm{~nm})$; small chain-like aggregates.

FIG. 2. PM collected on ELPI stage 3 (100-160 nm); medium fractal-like aggregates.
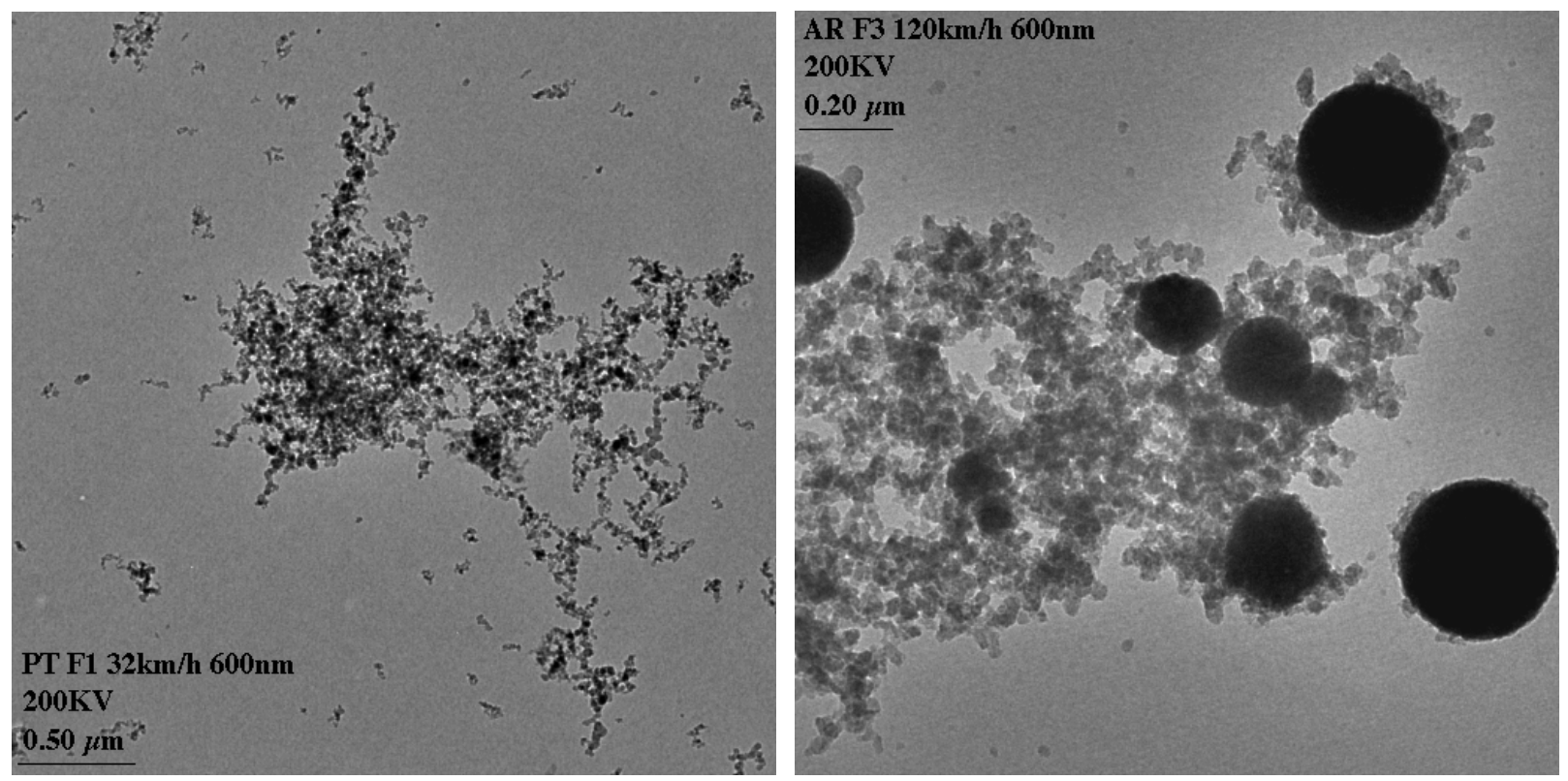

FIG. 3. PM collected on ELPI stage $7(600-1000 \mathrm{~nm})$; big sponge-like aggregates FIG. 4. PM collected on ELPI stage 7; particles from the aggregates (ca. $200 \mathrm{~nm}$ ). 